\title{
Screw dislocation core structure in the paramagnetic state of bcc iron from first-principles calculations
}

\author{
Luis Casillas-Trujillo, ${ }^{1, *}$ Davide Gambino $\odot,{ }^{1}$ Lisa Ventelon, ${ }^{2}$ and Björn Alling $\oplus^{1}$ \\ ${ }^{1}$ Department of Physics, Chemistry and Biology (IFM), Linköping University, 58183 Linköping, Sweden \\ ${ }^{2}$ Université Paris-Saclay, CEA, Service de Recherches de Métallurgie Physique, 91191 Gif-sur-Yvette, France
}

(Received 4 June 2020; revised 3 August 2020; accepted 27 August 2020; published 16 September 2020)

\begin{abstract}
Iron-based alloys are widely used as structural components in engineering applications. This calls for a fundamental understanding of their mechanical properties, including those of pure iron. Under operational temperatures the mechanical and magnetic properties will differ from those of ferromagnetic body-centeredcubic iron at $0 \mathrm{~K}$. In this theoretical work we study the effect of disordered magnetism on the screw dislocation core structure and compare with results for the ordered ferromagnetic case. Dislocation cores control some local properties such as the choice of glide plane and the associated dislocation mobility. Changes in the magnetic state can lead to modifications in the structure of the core and affect dislocation mobility. In particular, we focus on the core properties of the $\frac{1}{2}\langle 111\rangle$ screw dislocation in the paramagnetic state. Using the noncollinear disordered local moment approximation to address paramagnetism, we perform structural relaxations within density functional theory. We obtain the dislocation core structure for the easy and hard cores in the paramagnetic state, and compare them with their ferromagnetic counterparts. By averaging the energy of several disordered magnetic configurations, we obtain an energy difference between the easy- and hard-core configurations, with a lower, but statistically close, value than the one reported for the ferromagnetic case. The magnetic moment and atomic volume at the dislocation core differ between paramagnetic and ferromagnetic states, with possible consequences on the temperature dependence of defect-dislocation interactions.
\end{abstract}

DOI: 10.1103/PhysRevB.102.094420

\section{INTRODUCTION}

Iron and iron alloys, like steels, are the most technologically important metallic systems and have been so for millennia. As such, they are currently being used as structural materials in nuclear energy applications and they are also candidates for next generation nuclear technology [1]. In many of these applications they are subjected to high operational temperatures. Furthermore, new nuclear energy technologies impose even more demanding operational conditions [2]. Structural materials must possess high mechanical stability to reliably withstand these conditions, thus an understanding of the deformation mechanisms at high temperatures is needed. The plastic behavior in body-centered-cubic (bcc) iron is attributed to screw dislocation core effects. Indeed, the dislocation core controls some local properties such as the choice of glide plane and the associated dislocation mobility [3]. Meanwhile, high temperature increases magnetic disorder, and when the critical temperature is reached, $1043 \mathrm{~K}$ in the case of bcc iron, the system no longer exhibits any long-range magnetic ordering, although the local spin polarization of the atoms is still present. The bond strength between

\footnotetext{
*Corresponding author: luis.casillas.trujillo@liu.se
}

Published by the American Physical Society under the terms of the Creative Commons Attribution 4.0 International license. Further distribution of this work must maintain attribution to the author(s) and the published article's title, journal citation, and DOI. Funded by Bibsam. ordered and disordered magnetic states is different and thus magnetic disorder may alter the dislocation core properties and impact plastic behavior. On the other hand, changes in mechanical properties with temperature might also originate in, e.g., lattice vibrations, structural transitions, and alloy configurational changes. These are all present in experiments and are difficult to separate. To add further complexity, the magnetic state and lattice vibrations are coupled at high temperature $[4,5]$. In order to gain the detailed fundamental understanding needed to design mechanical properties of novel alloys, theoretical work that can pinpoint the specific effects of magnetic disorder is important.

The simulation of the paramagnetic state by first-principles calculations is challenging and the paramagnetic state is often approximated by the nonmagnetic state, but this approach can lead to misleading results $[6,7]$. In order to properly describe the paramagnetic state, we employ the disordered local moment approach (DLM) [8] as implemented in a supercell framework $[9,10]$. Two types of core configuration for the $\frac{1}{2}\langle 111\rangle$ screw dislocation in bcc metals can be obtained by centering the dislocation in between three $\langle 111\rangle$ atomic columns [11], namely the easy core, where the chirality of the three $\langle 111\rangle$ atomic columns that compose the core is reversed compared to the bulk bcc structure, and the hard core, in which the three columns that compose the core lie at the same altitude in the $\langle 111\rangle$ direction. There exists a third position of high symmetry, when the dislocation is centered on an atomic column and this position is approached by placing the dislocation in the vicinity of the atomic column and it is referred as the split core [12]. In pure bcc metals, density functional 
theory (DFT) calculations showed that the easy-core structure is the minimum-energy stable position for the dislocation while the hard core is an unstable position [13-19]. Dislocation glide in bec metals occurs via a thermally activated displacement between two stable easy-core positions along a $\{110\}$ plane with a corresponding single-humped Peierls barrier and a dislocation trajectory that deviates from the straight path between equilibrium configurations [20,21].

Ferromagnetism has been stated to play a fundamental role in the stabilization of the easy core in bcc iron, with nonmagnetic or antiferromagnetic configurations being unstable [22]. However, nonmagnetic or antiferromagnetic bcc iron are of no practical interest, and the situation in the paramagnetic state, such as alpha iron and delta iron above the transition temperature, is unknown. Iron also displays a distinct energy landscape from other bcc metals, where in particular DFT calculations evidenced a monkey saddle type of energy extremum for the hard core, instead of a local maximum as in all other bcc metals [13]. The magnetic state has an impact on point defect formation energies as recent studies have shown that there exists a difference in point defect formation energy between the ferromagnetic and paramagnetic regimes. Vacancy formation energy in bcc iron using DFT [23] and DFT+dynamical mean field theory (DMFT) [24] showed a $0.5 \mathrm{eV}$ difference between the ferromagnetic and paramagnetic states, where $\sim 0.1 \mathrm{eV}$ of the difference originated in geometric relaxation differences between the two magnetic states. For carbon interstitial atoms in octahedral positions, a difference in formation energy of $0.27 \mathrm{eV}$ was found out, of which more than half was shown to be due to differences in the relaxed positions in the two magnetic states [23]. Thus, in addition to directly impacting the dislocation core, the magnetic state can alter the interaction of point defects with dislocations and lead to different solute segregation profiles.

The screw dislocation core in ferromagnetic bcc iron has been extensively studied using first-principles calculations [13-15,20,25-36], but no assessment of the effect of magnetic disorder on the dislocation core has been performed. As such, the development of theoretical methods that assess the effect of magnetic disorder on plastic properties and dislocation mediated mechanical properties allow new insights in the physics of magnetostructural interactions via the studied coupling between spin disorder, dislocation core structure, and local magnetic moments. In this work, we study the impact of disordered magnetism on the core structure of the $\frac{1}{2}\langle 111\rangle$ screw dislocation in bcc iron. We investigate the structure for the easy- and hard-core configurations in the paramagnetic state, and the energy difference between them, and compare and contrast with those in the ferromagnetic phase. We also study the local magnetic moments and Voronoi atomic volumes and compare them in the two magnetic states.

\section{METHODS}

\section{A. DFT calculations}

A dislocation dipole is introduced in the simulation box in a quadrupolar arrangement, which allows for the use of triperiodic boundary conditions [3]. The dislocation is placed in the center of gravity of three neighboring $\langle 111\rangle$ atomic
TABLE I. Ab initio lattice and elastic constants of bcc Fe.

\begin{tabular}{lc}
\hline \hline & Ferromagnetic \\
\hline Lattice constant $(\AA)$ & 2.83 \\
$C_{11}(\mathrm{GPa})$ & 276 \\
$C_{12}(\mathrm{GPa})$ & 136 \\
$C_{44}(\mathrm{GPa})$ & 103 \\
\hline \hline
\end{tabular}

columns. Depending on the sign of the Burgers vector, the easy- or hard-core configurations can be obtained [11]. Since the hard core is unstable, the coordinates along the Burgers vector direction of the three $\langle 111\rangle$ atomic columns composing the dislocation core are fixed to prevent the hard core from collapsing towards the neighboring easy core. The dislocation dipole is introduced into the simulation cell by applying the displacement field of each dislocation as given by anisotropic linear elasticity using the Babel program [37]. We use $a b$ initio calculated lattice and elastic constants to construct the dislocated crystal, which are shown in Table I. The atomic positions are relaxed with $a b$ initio calculations to minimize the energy of the simulation box using fixed periodicity vectors. The starting point for the relaxation is a relaxed screw dislocation structure in ferromagnetic iron. We use the ferromagnetic structure as a starting point; since the lattice constant has an effect on the core structure of the screw dislocation as reported by Yang et al. in bcc tantalum [38], the larger $2.89 \AA$ paramagnetic lattice constant [39] would make it difficult to separate the effect of magnetic disorder, which is our aim, from those of thermal expansion. The disordered local moment (DLM) approach is applied on top of this relaxed structure.

The DFT calculations are performed using the projector augmented wave pseudopotential scheme [40] as implemented in the Vienna Ab-initio Simulation Package (VASP) $[41,42]$ with the Perdew-Burke-Ernzenhof generalized gradient approximation [43] to model the exchange-correlation effects. A pseudopotential without semicore electrons is used for Fe. All calculations are performed using a 400-eV kineticenergy cutoff, a Methfessel-Paxton $0.2-\mathrm{eV}$ broadening, and with a convergence criterion on all forces of $0.01 \mathrm{eV} / \AA$ for structural relaxations. We use cells with a length in the $\langle 111\rangle$ direction of 1,2 , and 3 Burgers vectors $(b)$ leading to cells containing 135,270 , and 405 atoms respectively. $1 \times 2 \times 16$, $1 \times 2 \times 8$, and $1 \times 2 \times 6 k$-point grids were used for the $1-, 2$-, and $3-b$ cells respectively. Unless otherwise specified, we used the $1-b$ cells. Simulation cells contain a single dipole in a quadrupolar arrangement and the cell dimensions in the (111) plane perpendicular to $b$ are large enough to yield a converged dislocation core energy difference between the easy- and hardcore configurations [25]. The elastic constants are obtained from the strain-stress relationship as implemented in VASP using a unit cell of Fe with a $21 \times 21 \times 21 k$-point grid.

\section{B. DLM approach}

The real magnetization density in many magnetic materials can be described in terms of magnetic moments localized close to the atoms. The paramagnetic case can be viewed as a disordered distribution of such local moments. The 


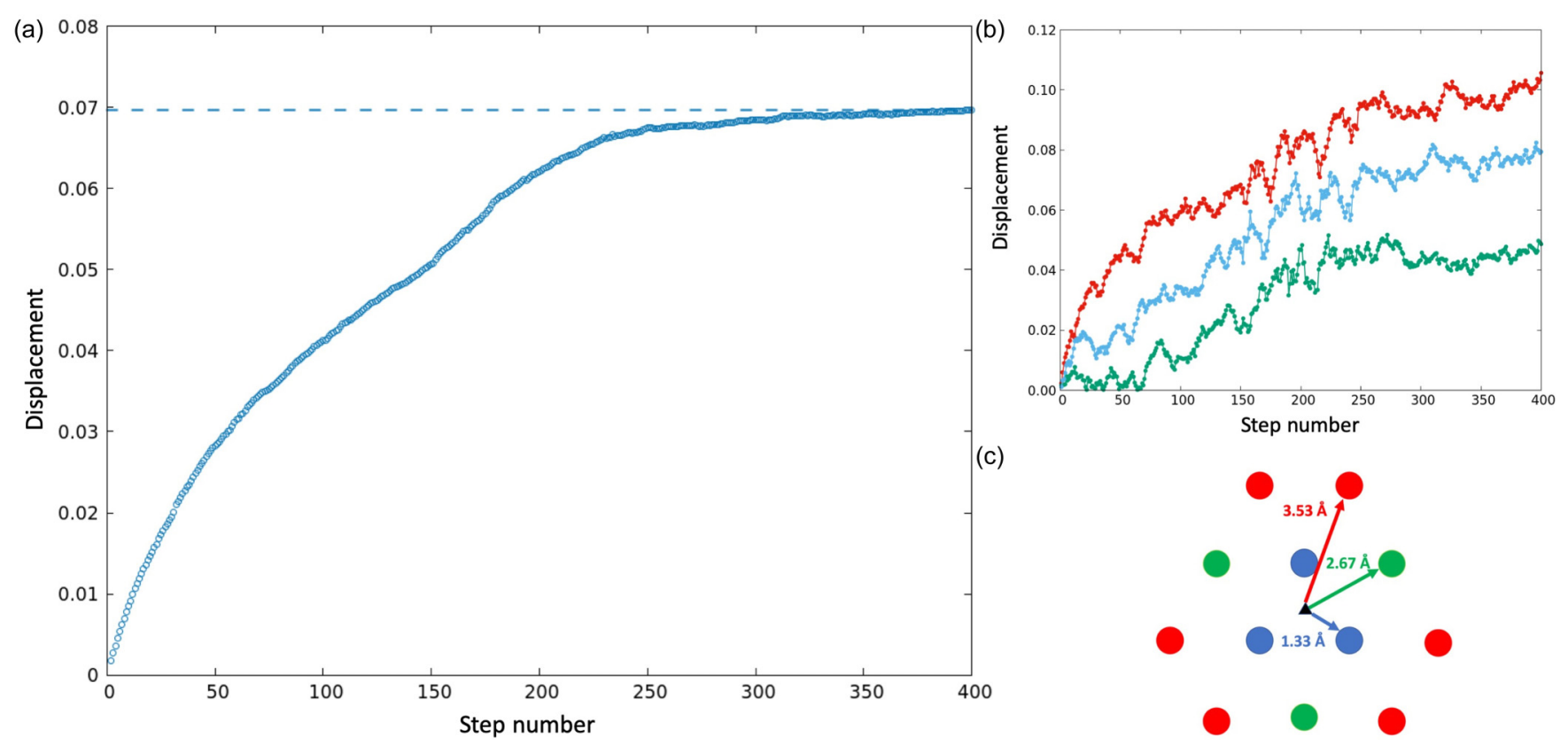

FIG. 1. (a) Cumulative average displacement as a function of simulation step number. (b) Cumulative displacement of the atomic positions close to the dislocation core averaged between the equivalent positions around the core. (c) Schematic representation of the atomic positions around the dislocation core.

fully disordered magnetic state corresponds to the paramagnetic limit where no long- or short-range magnetic order exists, approximating the state above the Curie temperature $\left(T_{\mathrm{C}}\right)$ and becoming a gradually better approximation with increasing temperature. Although no long-range order exists, some short-range order is still present above the transition temperature [44-46]. Körmann et al. [5] demonstrated in a study of vibrational properties of bcc Fe that the effect of temperature-dependent gradual magnetic disorder could be modeled from the knowledge of the ferromagnetic (FM) and DLM limits and combining them with the temperaturedependent short-range-order parameter. The direction of the magnetic moments quickly changes in time compared to the jump rates of atoms. This imposes challenges for modeling when atoms move from their high-symmetry positions such as in lattice relaxations. Lattice relaxations in bcc iron in the paramagnetic state have been commonly performed with ferromagnetic moments [24,46-48]. The interatomic bond strengths can greatly differ between the two magnetic states [49], leading to different atomic positions after relaxation. The DLM approach allows us to formulate a consistent electronicstructure based thermodynamic theory that accounts for the interplay between the configurational and magnetic degrees of freedom [50]. In the DLM simulations, the direction of the magnetic moments is randomly assigned, and can be implemented in a supercell approach $[9,10]$. In the case of $1-b$ supercells, the random configurations produce in-plane short-range-order (SRO) parameters close to zero, while it suffers from periodicity along the shorter dislocation line direction, where the periodic images form part of the nearestneighbor atom set. SRO values for $2-b$ and $3-b$ supercells are close to zero. The periodicity along the dislocation line direction also produces a ferromagnetic component, since the periodic images have the same magnetic-moment direction. We follow the relaxation procedure proposed by Gambino and Alling [23] for the paramagnetic state, which uses the DLM approach within DFT, where atoms are partially allowed to relax according to different disordered magnetic configurations in sequence until a steady displacement of the atoms from the initial positions is achieved. Equilibrium positions of each atom in the paramagnetic state are obtained by averaging the atomic positions of several configurations once the relaxation is converged. We use noncollinear spin-polarized calculations, and we constrain the direction of the magnetic moments using the method developed by $\mathrm{Ma}$ and Dudarev [51]. Figure 1(a) shows the convergence criteria to achieve a relaxed dislocation. We consider a relaxed structure when the cumulative displacement averaged over all atoms in the supercell with respect to the initial positions reaches a plateau and fluctuates around a constant value. We also check the displacements of the atoms near the dislocation core since they possess the largest displacements in the system. This is shown in Fig. 1(b), where the cumulative displacement for the core atoms also fluctuates around a constant value. The colors in Fig. 1(b) match the atomic positions displayed in Fig. 1(c).

\section{Magnetic sampling method}

The magnetic sampling method (MSM) approach [10] consists of performing static calculations using a large set of random magnetic moment configurations. With this approach the paramagnetic state can be modeled and a property of interest can be obtained as the arithmetic average over all the used configurations. The error of the mean value $P\left(\sigma_{\bar{P}}\right)$ of the desired property is calculated as the standard error 

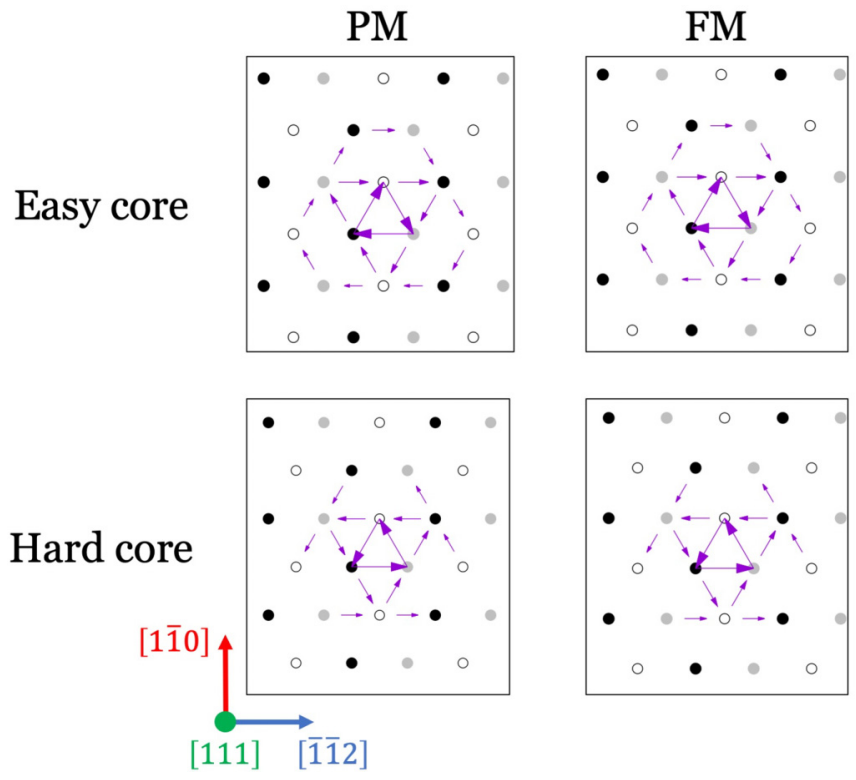

FIG. 2. DD maps of the easy- and hard-core configurations for the ferromagnetic and DLM relaxed paramagnetic dislocations.

$\pm 2 \sigma_{\bar{P}}=2 \sigma_{P} / \sqrt{N}$ giving a confidence interval of $95 \%$, where $N$ is the number of employed configurations.

\section{RESULTS}

\section{A. Dislocation core structure}

The dislocation core structure can be visualized using differential displacement (DD) maps [52]. Figure 2 shows the DD maps of the easy- and hard-core configurations relaxed in the paramagnetic state and in the ferromagnetic state. The DD maps show no substantial change between paramagnetic and ferromagnetic states, thus magnetic disorder does not affect the structure of the dislocation core. It should be noted that the hard core is stabilized in the paramagnetic state in our DLM calculations, while in the ferromagnetic state, the hard core can be stabilized only by fixing the coordinates of the core atoms along the Burgers vector direction.

Due to the periodic boundary conditions, the magnetic configuration is also repeated in space. This effect is accentuated along the dislocation line direction since the simulation cell size is short in this direction. As mentioned before, the periodic images of each atomic position are part of their nearest-neighbor set; as such they possess the same magnetic moment direction. To assess this periodicity issue, we use larger cells along the $\langle 111\rangle$ direction with $2-b$ and $3-b$ cell heights. We take the relaxed positions from the $1-b$ case, doubling or tripling the cell along the dislocation line direction. We use these cells as the starting point for new paramagnetic relaxations. For both cases, after further relaxation steps, the average displacement is $0.009 \AA$, with very small displacements of the atoms localized close to the core as for the 1- $b$ relaxation. These results indicate that the periodicity of the magnetic moments due to the short $1-b$ cell size has no considerable effect on the dislocation core relaxation.

\section{B. Energy}

From the relaxed dislocation structure in the paramagnetic state, we use the MSM approach by calculating the energy of 148 different magnetic configurations for both the easyand hard-core configurations. In Fig. 3 we plot the energy of each of the configurations and the cumulative average of

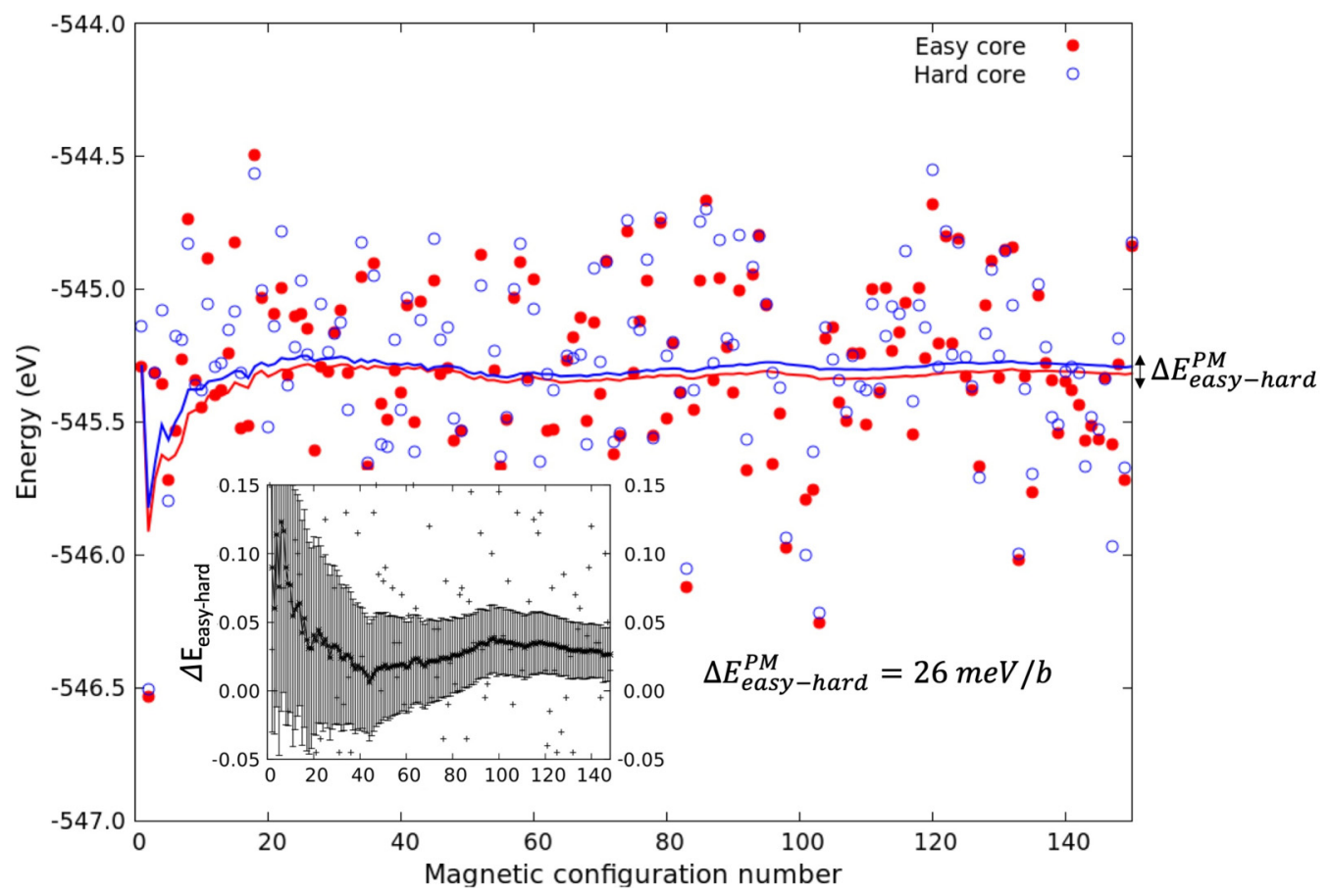

FIG. 3. Energies, accumulated mean energies, and energy difference between the easy and hard cores in the paramagnetic state for the different disordered magnetic configurations used in the MSM that together make up our model for the paramagnetic state. 

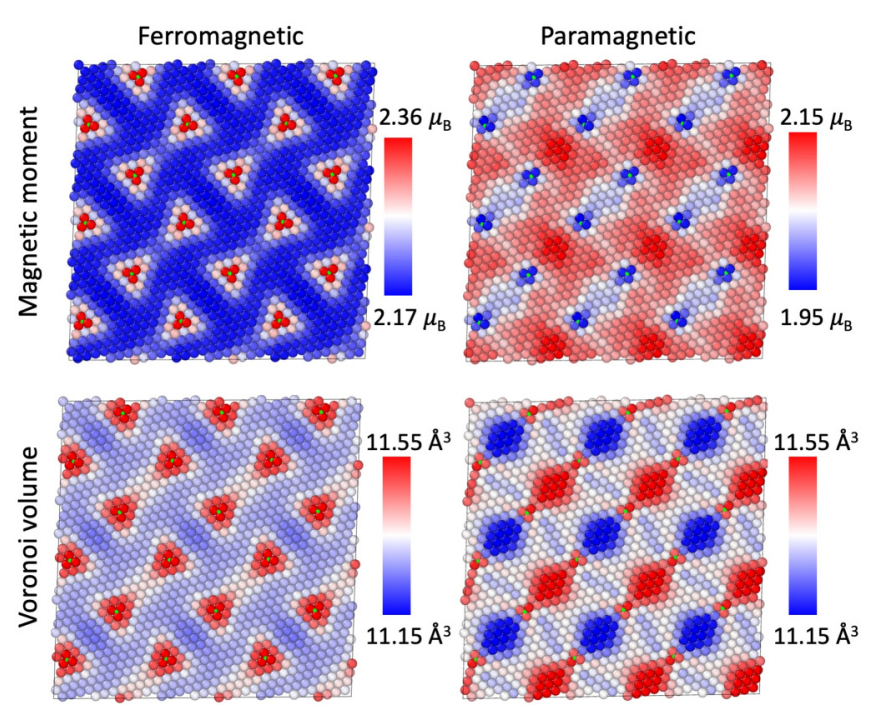

FIG. 4. Local magnetic moments and Voronoi volumes in the ferromagnetic and paramagnetic configurations for the easy core. the energies. For each configuration identification number in the $x$ axis of Fig. 3, we employ the most similar starting magnetic configuration that can be obtained for both the easyand hard-core configurations to minimize the stochastic noise in core energy differences. We find that in the paramagnetic state, approximated with the average of our sampled magnetic configurations, the easy core has a lower energy than the hard core. The average energy difference is $26 \pm 20 \mathrm{meV} / \mathrm{b}$ where the error bars correspond to a $95 \%$ confidence interval. With $92 \%$ confidence, the energy difference in the paramagnetic state is lower than the value we obtain for the ferromagnetic case of $40 \mathrm{meV} / \mathrm{b}$; with this lower value one might expect an increase in dislocation mobility in the paramagnetic as compared to the ferromagnetic state caused by magnetic effects (the FM value in agreement with the DFT value reported by Lüthi et al. [53]). In the inset of Fig. 3, we plot the average energy difference between the easy and hard cores. We note that $36 \%$ of the employed magnetic configurations yielded a lower energy for the hard core than for the easy core, which may indicate that some particular magnetic environments might be more favorable in the hard-core configuration.
Easy core

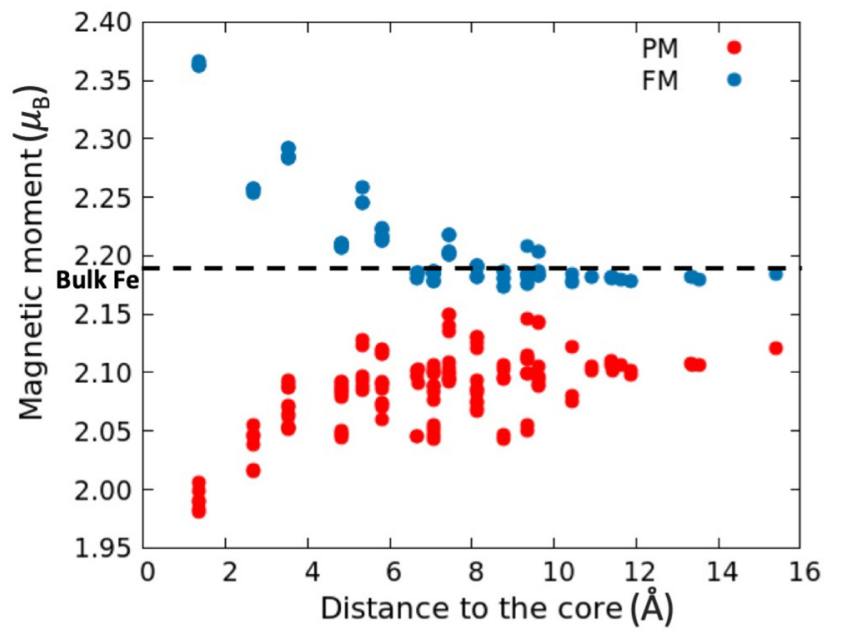

Easy core

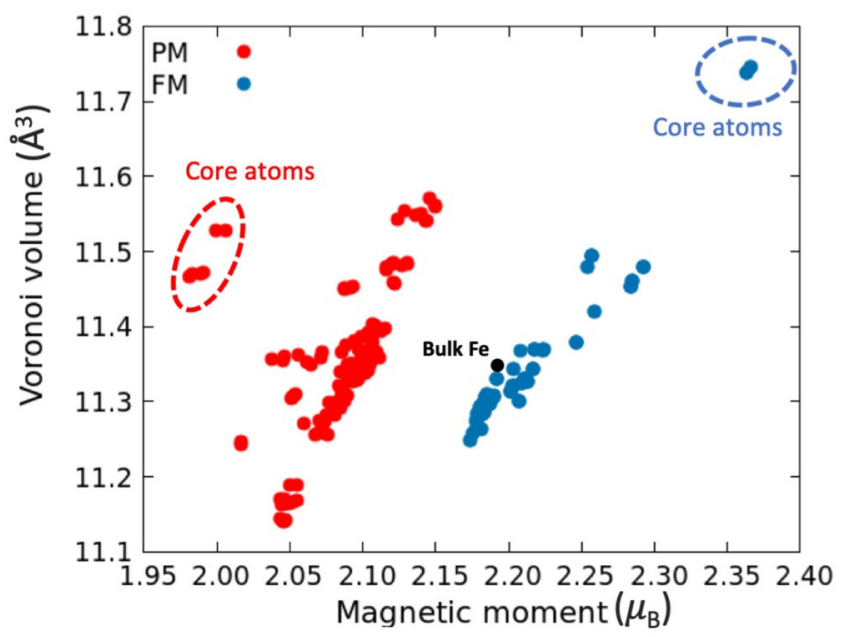

Hard core

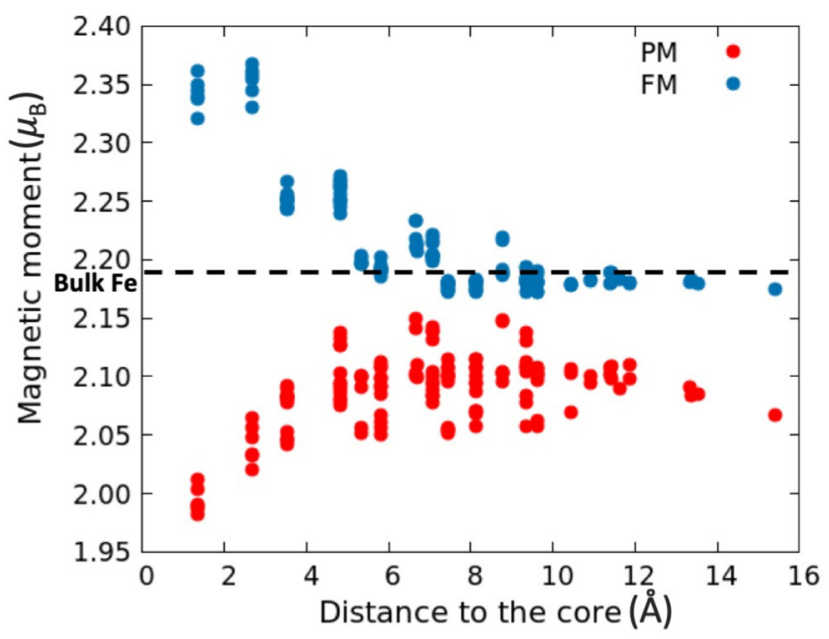

Hard core

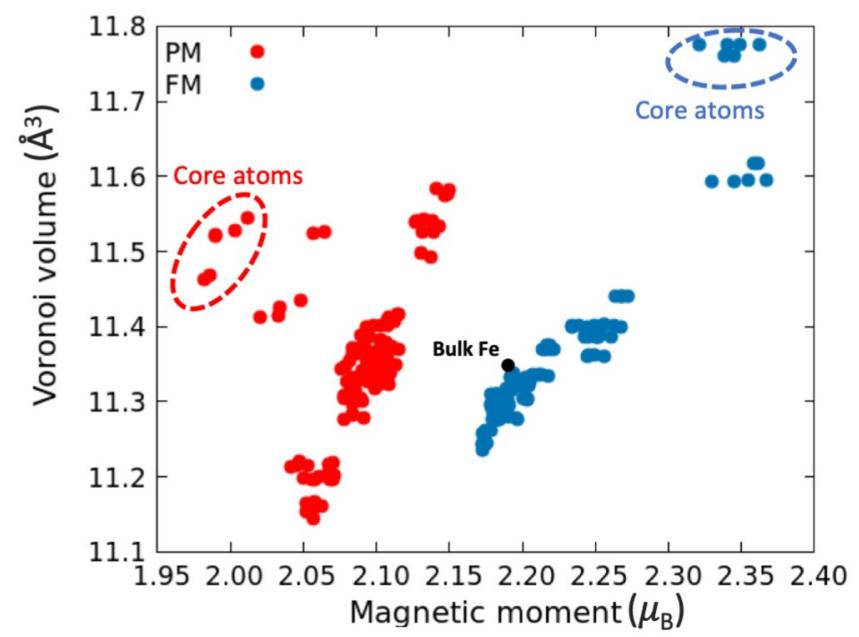

FIG. 5. Upper: Magnetic moment as a function of distance from the dislocation core for the easy- and hard-core configurations. Lower: Voronoi volume vs magnetic moment for the easy- and hard-core configurations. 


\section{Magnetic moments and atomic volumes}

The local magnetic moments are disturbed by the presence of the screw dislocation. In the ferromagnetic state, the magnetic moments of the atoms closest to the dislocation core present an average increase of $0.17 \mu_{\mathrm{B}}$ with respect to the bulk value of $2.19 \mu_{\mathrm{B}}$ in the case of the easy core, and of $0.15 \mu_{\mathrm{B}}$ in the case of the hard core, in close agreement with the values reported in the literature [30,54]. A comparison of the local magnetic-moment landscape between the paramagnetic and ferromagnetic regime is shown in the upper part of Fig. 4 in the case of the easy-core configuration. To obtain this landscape in the paramagnetic case, we average the magnitude of the output magnetic moments of different magnetic configurations of the relaxed structure. It can be observed that this landscape is different from the one displayed in ferromagnetic iron. A notable feature is that in the paramagnetic state, the lowest magnitude of the local magnetic moments occurs close to the dislocation core contrary to the ferromagnetic case where the opposite is observed. This is highlighted in the upper part of Fig. 5 where we plot the local magnetic moment versus distance from the dislocation core for both the easy- and hard-core configurations. In the ferromagnetic case, for both cores, the magnitude of the local magnetic moment increases as the atoms get closer to the core, while in the paramagnetic case the opposite is observed, i.e., the local magnetic moment decreases as the atoms get closer to the core. In Fig. 5 we have included the values for bulk FM Fe as a reference.

We also calculate the Voronoi atomic volumes, as shown in the lower portion of Fig. 4. The atomic volume landscape in the paramagnetic state also presents a distribution different from the ferromagnetic state distribution. Although the deviation from the bulk atomic value of $11.35 \AA^{3}$ is small, the dilatation and compression effect can be evaluated. In the ferromagnetic case, the magnetic moments under compression are reduced while those in dilatation are enhanced as is typical of the relationship between magnetic moment and volume (the so-called magnetovolume effect [55-57]). The lower portion of Fig. 5 better contrasts the behaviors of the ferromagnetic and paramagnetic phases, in which we plot the magnetic moment versus Voronoi volume. In general, both magnetic configurations follow the larger volume-larger moment trend, but it is possible to observe that the highlighted dislocation core positions in the paramagnetic state do not follow this relationship, having the smallest magnetic moment.

\section{DISCUSSION}

We have determined core structure and energy difference between easy and hard cores in the high-temperature paramagnetic limit and in the low-temperature FM limit. The present results can thus be used as a starting point to address the magnetic contributions at finite temperatures. Temperature dependence can be accounted for by the adiabatic coupling parameter $\alpha(T)$ proposed by Körmann et al. [5], who successfully used it to explain experimental observations of the temperature dependence of phonon frequencies in bcc Fe. In this approximation our results provide the ferromagnetic and paramagnetic limits, while finite temperature SRO can be

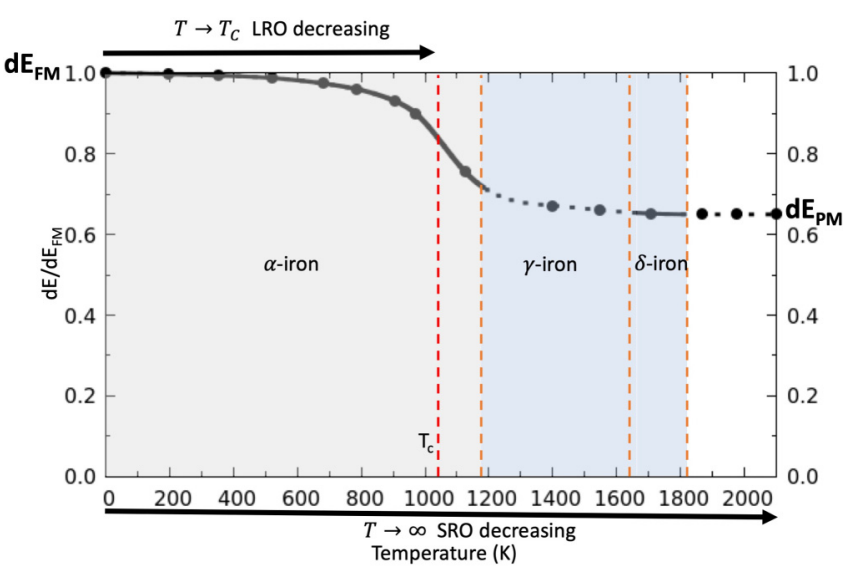

FIG. 6. Schematic representation of the $E_{\text {easy-hard }}$ temperature dependence in terms of its value in the FM low-temperature limit.

obtained with quantum Monte Carlo calculations. In Fig. 6, we have sketched the temperature dependence of $E_{\text {easy-hard }}$ by taking the temperature dependence of magnetic SRO from [5] and using our 1- $b$ calculations to approximate the hightemperature limit. The figure shows that the most rapid change in $d E$ takes place at temperatures around the Curie temperature. At $T_{\mathrm{C}}$ the value of $d E$ is 0.84 of the FM value. At the alpha-to-gamma transition temperature $1185 \mathrm{~K}\left(1.14 T_{\mathrm{C}}\right) d E$ is 0.72 of the FM value. In the high-temperature bcc delta phase, stable between 1667 and $1811 \mathrm{~K}\left(1.60-1.74 T_{\mathrm{C}}\right)$ the value of $d E$ is already extremely close to the approximation for the high-temperature PM limit of 0.65 . We want to emphasize that the contributions from lattice vibrations need to also be included for a full understanding of the temperature trend of the dislocation core energetics of bcc Fe.

\section{CONCLUSIONS}

In this work we assess the effect of magnetic disorder on the core structure of a $\frac{1}{2}\langle 111\rangle$ screw dislocation in bcc iron, by relaxing the easy- and hard-core configurations in the paramagnetic state using the DLM approach. We found that the difference in the structure of the dislocation core introduced by the magnetic disorder of the paramagnetic state is small compared to the ferromagnetic state for both the easy- and hard-core configurations. We employ the magnetic sampling method to obtain the energy of the easy- and hard-core configurations, and find that in the paramagnetic state the easy core has a lower energy than the hard core with an average energy difference between easy and hard cores of $26 \pm 20 \mathrm{meV} / \mathrm{b}$. This value is smaller than the value of $40 \mathrm{meV} / \mathrm{b}$ obtained in the ferromagnetic state but the large statistical error bars make it difficult to draw definite conclusions. Although the core structure is similar, there exists a change in the local magnetic moment landscape between the two magnetic states. We find a different behavior in the magnetic moment-volume relationship: in the ferromagnetic state, the larger the volume is, the larger the magnetic moment is while in the paramagnetic state this is no longer the case close to the dislocation core, with the smallest magnetic moment magnitude obtained close to the core. This could introduce a different behavior in the interaction of the dislocation with impurity atoms, in particular 
magnetic impurity atoms like $\mathrm{Cr}$ [58]. As the explicit effects of high-temperature magnetic disorder is not dramatic, the vibrational contributions must also be included to assess the explicit temperature dependent trend of dislocation properties in future studies.

\section{ACKNOWLEDGMENTS}

We want to thank L. Dezerald, D. Trinkle, and V. Razumovskiy for useful discussions. This research was carried out using computational resources provided by the National Supercomputer Center (NSC) in Linköping and the Swedish
National Infrastructure for Computing (SNIC). B.A. acknowledges financial support from the Swedish Research Council (VR) through International Career Grants No. 2014-6336 and No. 2019-05403, by Marie Sklodowska Curie Actions, Cofund, Project INCA 600398, from the Swedish Government Strategic Research Area in Materials Science on Functional Materials at Linköping University (Faculty Grant SFOMatLiU No. 2009 00971), from the Knut and Alice Wallenberg Foundation (Wallenberg Scholar Grant No. KAW2018.0194), as well as support from the Swedish Foundation for Strategic Research through the Future Research Leaders 6 program, FFL 15-0290.
[1] P. Yvon and F. Carré, Structural materials challenges for advanced reactor systems, J. Nucl. Mater. 385, 217 (2009).

[2] S. J. Zinkle and J. T. Busby, Structural materials for fission \& fusion energy, Mater. Today 12, 12 (2009).

[3] D. Rodney, L. Ventelon, E. Clouet, L. Pizzagalli, and F. Willaime, $A b$ initio modeling of dislocation core properties in metals and semiconductors, Acta Mater. 124, 633 (2017).

[4] B. Alling, F. Körmann, B. Grabowski, A. Glensk, I. A. Abrikosov, and J. Neugebauer, Strong impact of lattice vibrations on electronic and magnetic properties of paramagnetic Fe revealed by disordered local moments molecular dynamics, Phys. Rev. B 93, 224411 (2016).

[5] F. Körmann, B. Grabowski, B. Dutta, T. Hickel, L. Mauger, B. Fultz, and J. Neugebauer, Temperature Dependent MagnonPhonon Coupling in bcc Fe from Theory and Experiment, Phys. Rev. Lett. 113, 165503 (2014).

[6] B. Alling, T. Marten, and I. A. Abrikosov, Questionable collapse of the bulk modulus in CrN, Nat. Mater. 9, 283 (2010).

[7] F. Rivadulla, M. Bañobre-López, C. X. Quintela, A. Piñeiro, V. Pardo, D. Baldomir, M. A. López-Quintela, J. Rivas, C. A. Ramos, H. Salva, J.-S. Zhou, and J. B. Goodenough, Reduction of the bulk modulus at high pressure in $\mathrm{CrN}$, Nat. Mater. 8, 947 (2009).

[8] B. L. Gyorffy, A. J. Pindor, J. Staunton, G. M. Stocks, and H. Winter, A first-principles theory of ferromagnetic phase transitions in metals, J. Phys. F 15, 1337 (1985).

[9] B. Alling, Theory of the ferromagnetism in Ti1-xCrxN solid solutions, Phys. Rev. B 82, 054408 (2010).

[10] B. Alling, T. Marten, and I. A. Abrikosov, Effect of magnetic disorder and strong electron correlations on the thermodynamics of CrN, Phys. Rev. B 82, 184430 (2010).

[11] V. Vitek, Theory of the core structures of dislocations in bodycentered-cubic metals, Cryst. Lattice Defects 5(1), 1 (1974).

[12] S. Takeuchi, Core structure of a screw dislocation in the BCC lattice and its relation to slip behaviour of $\alpha$-iron, Philos. Mag. A 39, 661 (1979).

[13] L. Dezerald, L. Ventelon, E. Clouet, C. Denoual, D. Rodney, and F. Willaime, $A b$ initio modeling of the two-dimensional energy landscape of screw dislocations in bcc transition metals, Phys. Rev. B 89, 024104 (2014).

[14] S. L. Frederiksen and K. W. Jacobsen, Density functional theory studies of screw dislocation core structures in bec metals, Philos. Mag. 83, 365 (2003).
[15] S. Ismail-Beigi and T. Arias, Ab Initio Study of Screw Dislocations in Mo and Ta: A New Picture of Plasticity in bcc Transition Metals, Phys. Rev. Lett. 84, 1499 (2000).

[16] D. Segall, A. Strachan, W. A. Goddard III, S. Ismail-Beigi, and T. Arias, $A b$ initio and finite-temperature molecular dynamics studies of lattice resistance in tantalum, Phys. Rev. B 68, 014104 (2003).

[17] L. Ventelon and F. Willaime, Core structure and Peierls potential of screw dislocations in $\alpha$-Fe from first principles: Cluster versus dipole approaches, J. Comput. Aided Mater. Des. 14, 85 (2007).

[18] C. R. Weinberger, G. J. Tucker, and S. M. Foiles, Peierls potential of screw dislocations in bcc transition metals: Predictions from density functional theory, Phys. Rev. B 87, 054114 (2013).

[19] C. Woodward and S. I. Rao, Flexible Ab Initio Boundary Conditions: Simulating Isolated Dislocations in bec Mo and Ta, Phys. Rev. Lett. 88, 216402 (2002).

[20] L. Dezerald, D. Rodney, E. Clouet, L. Ventelon, and F. Willaime, Plastic anisotropy and dislocation trajectory in BCC metals, Nat. Commun. 7, 11695 (2016).

[21] R. Gröger and V. Vitek, Determination of positions and curved transition pathways of screw dislocations in bcc crystals from atomic displacements, Mater. Sci. Eng., A 643, 203 (2015).

[22] K. Odbadrakh, G. Samolyuk, D. Nicholson, Y. Osetsky, R. Stoller, and G. Stocks, Decisive role of magnetism in the interaction of chromium and nickel solute atoms with $1 / 2\langle 111\rangle$ screw dislocation core in body-centered cubic iron, Acta Mater. 121, 137 (2016).

[23] D. Gambino and B. Alling, Lattice relaxations in disordered Febased materials in the paramagnetic state from first principles, Phys. Rev. B 98, 064105 (2018).

[24] P. Delange, T. Ayral, S. I. Simak, M. Ferrero, O. Parcollet, S. Biermann, and L. Pourovskii, Large effects of subtle electronic correlations on the energetics of vacancies in $\alpha$-Fe, Phys. Rev. B 94, 100102 (2016).

[25] E. Clouet, L. Ventelon, and F. Willaime, Dislocation Core Energies and Core Fields from First Principles, Phys. Rev. Lett. 102, 055502 (2009).

[26] E. Clouet, L. Ventelon, and F. Willaime, Dislocation core field. II. Screw dislocation in iron, Phys. Rev. B 84, 224107 (2011).

[27] A. Hishinuma, A. Kohyama, R. Klueh, D. Gelles, W. Dietz, and K. Ehrlich, Current status and future R\&D for reducedactivation ferritic/martensitic steels, J. Nucl. Mater. 258-263, 193 (1998). 
[28] M. Hossain and J. Marian, Stress-dependent solute energetics in W-Re alloys from first-principles calculations, Acta Mater. 80, 107 (2014).

[29] M. Itakura, H. Kaburaki, and M. Yamaguchi, First-principles study on the mobility of screw dislocations in bcc iron, Acta Mater. 60, 3698 (2012).

[30] K. Odbadrakh, A. Rusanu, G. M. Stocks, G. D. Samolyuk, M. Eisenbach, Y. Wang, and D. M. Nicholson, Calculated electronic and magnetic structure of screw dislocations in alpha iron, J. Appl. Phys. 109, 07E159 (2011).

[31] L. Romaner, C. Ambrosch-Draxl, and R. Pippan, Effect of Rhenium on the Dislocation Core Structure in Tungsten, Phys. Rev. Lett. 104, 195503 (2010).

[32] L. Romaner, V. Razumovskiy, and R. Pippan, Core polarity of screw dislocations in Fe-Co alloys, Philos. Mag. Lett. 94, 334 (2014).

[33] G. D. Samolyuk, Y. Osetsky, and R. Stoller, The influence of transition metal solutes on the dislocation core structure and values of the Peierls stress and barrier in tungsten, J. Phys.: Condens. Matter 25, 025403 (2012).

[34] D. R. Trinkle and C. Woodward, The chemistry of deformation: How solutes soften pure metals, Science 310, 1665 (2005).

[35] L. Ventelon and F. Willaime, Generalized stacking-faults and screw-dislocation core-structure in bcc iron: a comparison between ab initio calculations and empirical potentials, Philos. Mag. 90, 1063 (2010).

[36] G. Wang, A. Strachan, T. Çağın, and W.A. Goddard III, Role of core polarization curvature of screw dislocations in determining the Peierls stress in bcc Ta: A criterion for designing high-performance materials., Phys. Rev. B 67, 140101 (2003).

[37] E. Clouet Babel, available at http://emmanuel.clouet.free.fr/ Programs/Babel.

[38] L. Yang, P. Söderlind, and J. Moriarty, Atomistic simulation of pressure-dependent screw dislocation properties in bcc tantalum, Mater. Sci. Eng., A 309, 102 (2001).

[39] I. Seki and K. Nagata, Lattice constant of iron and austenite including its supersaturation phase of carbon, ISIJ Int. 45, 1789 (2005).

[40] P. E. Blöchl, Projector augmented-wave method, Phys. Rev. B 50, 17953 (1994).

[41] G. Kresse and J. Furthmüller, Efficiency of ab-initio total energy calculations for metals and semiconductors using a plane-wave basis set, Comput. Mater. Sci. 6, 15 (1996).

[42] G. Kresse and J. Furthmüller, Efficient iterative schemes for ab initio total-energy calculations using a plane-wave basis set, Phys. Rev. B 54, 11169 (1996).
[43] J. P. Perdew, K. Burke, and M. Ernzerhof, Generalized Gradient Approximation Made Simple, Phys. Rev. Lett. 77, 3865 (1996).

[44] V. Antropov, Magnetic short-range order above the Curie temperature of Fe and Ni, Phys. Rev. B 72, 140406 (2005).

[45] F. Körmann, A. Dick, T. Hickel, and J. Neugebauer, Role of spin quantization in determining the thermodynamic properties of magnetic transition metals, Phys. Rev. B 83, 165114 (2011).

[46] A. V. Ruban and V. I. Razumovskiy, Spin-wave method for the total energy of paramagnetic state, Phys. Rev. B 85, 174407 (2012).

[47] H. Ding, V. I. Razumovskiy, and M. Asta, Self diffusion anomaly in ferromagnetic metals: A density-functional-theory investigation of magnetically ordered and disordered $\mathrm{Fe}$ and $\mathrm{Co}$, Acta Mater. 70, 130 (2014).

[48] N. Sandberg, Z. Chang, L. Messina, P. Olsson, and P. Korzhavyi, Modeling of the magnetic free energy of selfdiffusion in bcc Fe, Phys. Rev. B 92, 184102 (2015).

[49] F. Körmann, A. Dick, B. Grabowski, T. Hickel, and J. Neugebauer, Atomic forces at finite magnetic temperatures: Phonons in paramagnetic iron, Phys. Rev. B 85, 125104 (2012).

[50] I. A. Abrikosov, A. V. Ponomareva, P. Steneteg, S. A. Barannikova, and B. Alling, Recent progress in simulations of the paramagnetic state of magnetic materials, Curr. Opin. Solid State Mater. Sci. 20, 85 (2016).

[51] P.-W. Ma and S. Dudarev, Constrained density functional for noncollinear magnetism, Phys. Rev. B 91, 054420 (2015).

[52] V. Vítek, R. C. Perrin, and D. K. Bowen, The core structure of 1/2(111) screw dislocations in b.c.c. crystals, Philos. Mag. A 21, 1049 (1970).

[53] B. Lüthi, L. Ventelon, C. Elsässer, D. Rodney, and F. Willaime, First principles investigation of carbon-screw dislocation interactions in body-centered cubic metals, Modell. Simul. Mater. Sci. Eng. 25, 084001 (2017).

[54] L. Ventelon, F. Willaime, E. Clouet, and D. Rodney, Ab initio investigation of the Peierls potential of screw dislocations in bcc Fe and W, Acta Mater. 61, 3973 (2013).

[55] V. Iota, J.-H. P. Klepeis, C.-S. Yoo, J. Lang, D. Haskel, and G. Srajer, Electronic structure and magnetism in compressed $3 \mathrm{~d}$ transition metals, Appl. Phys. Lett. 90, 042505 (2007).

[56] U. Krauss and U. Krey, Local magneto-volume effect in amorphous iron, J. Magn. Magn. Mater. 98, L1 (1991).

[57] L. Zhang, M. Šob, Z. Wu, Y. Zhang, and G.-H. Lu, Characterization of iron ferromagnetism by the local atomic volume: from three-dimensional structures to isolated atoms, J. Phys.: Condens. Matter 26, 086002 (2014).

[58] A. Ponomareva, A. Ruban, O. Y. Vekilova, S. Simak, and I. Abrikosov, Effect of pressure on phase stability in $\mathrm{Fe}-\mathrm{Cr}$ alloys, Phys. Rev. B 84, 094422 (2011). 